\title{
Treino muscular inspiratório em idosos na ventilação mecânica: uma revisão sistemática
}

\section{Inspiratory muscle training in the elderly in mechanical ventilation: a systematic review}

\author{
Aurea Gonçalves Ferreira, Daniel Vicentini de Oliveira, Emmanuel Dias de Sousa \\ Lopes, Rodrigo Marques Tonella, Cláudia Regina Barbosa, Luiz Cláudio Martins
}

Como citar este artigo: FERREIRA, A. G.; OLIVEIRA, D. V.; LOPES, E. D. S.; TONELLA, R. M.; BARBOSA, C. R.; MARTINS, L. C.

Treino muscular inspiratório em idosos na ventilação mecânica: uma revisão sistemática. Revista Saúde (Sta. Maria). 2019; 45 (2).

\section{Autor correspondente:}

Nome: Aurea Gonçalves Ferreira E-mail: aurea_fisio@hotmail.com

Telefone: 19) 981645678

Formação Profissional: Formada

Fisioterapia e Mestra em

Cerontologia pela Faculdade de

Ciências Médicas (FCM),

Campinas. SP, Brasil.

Filiação Institucional: Faculdade Anhanguera Educacional

Endereço para correspondência:

Rua: Padre Guilherme Ary n: 76

Bairro: Vila Saturnia

Cidade: Campinas

Estado: São Paulo

CEP: | 3031 -090

Data de Submissão:

13/04/2019

Data de aceite:

02/07/2019

Conflito de Interesse: Não há conflito de interesse

\section{(cc) $\mathrm{BY}-\mathrm{NC}-\mathrm{ND}$}

\section{RESUMO}

Este estudo teve o objetivo de avaliar os artigos de treinamento muscular inspiratório em idosos com desmame da ventilação mecânica, nos últimos cinco anos. O levantamento bibliográfico foi realizado através de publicações indexadas na base de dados da Biblioteca Virtual de Saúde (BVS): Scientific Electronic Library Online (SCIELO), Literatura Latino-Americana em Ciências de Saúde (LILACS), Medical Literature Analysis and Retrieval System Online (MEDLINE), US National Library of Medicine National Institutesof Health (PUBMED), Controlled Trials database (COCHRANE) e Physiotherapy evidence database (PEDro). Para organização do estudo, foram seguidos os critérios da revisão sistemática. Após avaliação, foram selecionados cinco artigos que se enquadravam nos critérios do estudo. Os artigos incluídos na revisão apresentaram rigor metodológico em todos os protocolos de treino muscular inspiratório utilizado, sendo que nem todos eram específicos para idosos, porém a idade média encontrada dos participantes em todos os estudos estava acima dos 60 anos de idade, apresentado uma variação entre 60 e 81 anos. A presente revisão sistemática evidenciou a importância do treino muscular inspiratório em idosos auxiliando no desmame da ventilação mecânica.

PALAVRAS-CHAVE: Envelhecimento, Unidade de Terapia Intensiva, Desmame do respirador, Diafragma, Fisioterapia.

\section{ABSTRACT}

This study aim to evaluate inspiratory muscle training in the elderly with weaning from mechanical ventilation in the last five years. The literature review was carried out through publications indexed in the Virtual Health Library (VHL) database: Scientific Electronic Library Online (SCIELO), Latin American Literature in Health Sciences (LILACS), Medical Literature Analysis and Retrieval System Online (MEDLINE), National Library of Medicine National Institutes of Health (PUBMED), Controlled Trials database (COCHRANE) and Physiotherapy evidence database (PEDro). To organize the study, the criteria of the systematic review were followed. After evaluation, five articles were selected that fit the study criteria. The articles included in the review presented methodological rigor in all inspiratory muscle training protocols used, not all of which were specific to the elderly, but the mean age of the participants in all studies was above 60 years of age, with a variation between 60 and 81 years. This systematic review evidenced the importance of inspiratory muscle training in the elderly, assisting in the weaning of mechanical ventilation.

KEYWORDS: Aging, Intensive Care Units, Ventilator weaning, Respiratory Diaphragm, Phisiotherapy. 


\section{INTRODUÇÃO}

O envelhecimento populacional nos últimos anos, ocasionou aumento de internações hospitalares de idosos com necessidade de cuidados intensivos dentro de uma unidade de terapia intensiva. Assim, gerando questionamentos pertinentes sobre o tratamento deste tipo de paciente a cada dia e levantando preocupações aos cofres públicos e a saúde, devido ao aumento de internações hospitalares de idosos e muito idosos, consumindo cerca de $60 \%$ das diárias hospitalares no sistema único de saúde (SUS) ${ }^{1}$.

A internação de idosos dentro da unidade de terapia intensiva pode levar a complicações do quadro inicial do paciente devido ao quadro de fragilidade instalada e durante o envelhecimento ocorrem alterações importantes no sistema respiratório, causando mudanças estruturais e funcionais, podendo levar ao pulmão senil. O pulmão senil apresenta perda da propriedade de recuo elástico, tornando-se mais complacente em relação à parede torácica, mais rígida decorrente as alterações estruturais e alterações da pressão intratorácica. O idoso poderá apresentar alterações no sistema respiratório durante o envelhecimento associadas a comorbidades, levando ao aumento do tempo de internação hospitalar e necessitar do suporte ventilatório $0^{1,2}$.

Quando o idoso necessitar do suporte ventilatório por tempo prolongado, poderá apresentar comprometimento da sua capacidade pulmonar, maior tempo de internação, podendo desencadear síndrome do imobilismo no leito e sarcopenia. A necessidade de suporte ventilatório pode agregar a fatores de mortalidade por aumento do tempo de internação hospitalar e infecções acometidas neste idoso e irá precisar de acompanhamento da equipe multidisciplinar para auxiliar na melhora do quadro instalado $0^{3,4}$.

Diante disto, destaca-se o fisioterapeuta que faz parte da equipe multidisciplinar e auxilia o paciente durante o suporte ventilatório realizando o manejo ventilatório e o desmame da ventilação mecânica invasiva, como também pode realizar o treino muscular inspiratório (TMI), recrutando fibras musculares que levam ao fortalecimento do músculo diafragma. Este treino pode ser realizado com o auxílio de equipamentos que empregam cargas durante a inspiração 5 .

O TMI melhora a força muscular inspiratória, permitindo a evolução do paciente durante o desmame prolongado, porém alguns fatores podem interferir durante este treino como: hipoxemia, hipercapnia, diminuição da capacidade pulmonar total e vital, dificultando na evolução do treino. A escolha adequada do protocolo do treino e a prevenção tem como o principal objetivo em desenvolver um condicionamento muscular para que o paciente não apresente piora da fadiga muscular durante o treinamento ${ }^{7,8}$.

Um estudo relatou que o treino muscular inspiratório, melhora a qualidade de vida destes indivíduos e quando realizado por meio de dispositivos que impõem cargas resistivas, podem aumentar a pressão inspiratória máxima (PImáx) e possibilitar o desmame ventilatório ${ }^{6}$. 
O objetivo desta revisão sistemática da literatura, foi avaliar os artigos de treinamento muscular inspiratório em idosos em desmame da ventilação mecânica, identificar os tipos de protocolo de treinamento realizados nos últimos cinco anos e seus respectivos dispositivos.

\section{MÉTODO}

Identificação do estudo

Trata-se de uma revisão sistemática da literatura, a qual buscou sistematizar estudos quase-experimental e experimental, avaliando o comprometimento das pesquisas realizadas para agregar ao nosso estudo, dentro dos critérios de inclusão, segundo as pesquisas na prática baseada em evidencias (PBE) 9 .

Para organização do estudo, seguimos os critérios das diretrizes de revisão sistemática (PRISMA), realizando as etapas de critério de inclusão da seguinte forma: delimitação do tema, definição das bases de dados e seus descritores, extração dos dados analisados e tabulados ${ }^{10}$.

A delimitação do problema durante o levantamento do estudo, foi identificar nas principais bases bibliográficas como está sendo realizado nos últimos cinco anos o treino muscular inspiratório em idosos na ventilação mecânica. Buscamos em território nacional e internacional, identificar quais protocolos foram realizados e quais dispositivos de treino muscular inspiratório foram utilizados.

As principais bases bibliográficas utilizadas durante a pesquisa estavam indexadas na base de dados da Biblioteca Virtual de Saúde (BVS): Scientific Electronic Library Online (SCIELO), Literatura Latino-Americana em Ciências de Saúde (LILACS), Medical Literature Analysis and Retrieval System Online (MEDLINE), US National Library of Medicine National Institutesof Heath (PUBMED), Controlled Trials database (COCHRANE) e Physiotherapy evidence database (PEDro).

Características dos artigos integrados ao estudo

Os pesquisadores analisaram de forma padronizada com os seguintes critérios de inclusão: Artigos nacionais e internacionais, publicações realizadas nos anos de 2014 a 2018. Os descritores incluídos para a pesquisa foram: Idosos, Unidade de Terapia Intensiva, Desmame do respirador, diafragma e fisioterapia. Foram incluídos estudos em pacientes idosos com 60 anos ou mais, em ventilação mecânica invasiva, em processo de desmame e que foram realizados protocolos de treino muscular inspiratório com dispositivos de limite de pressão. 
Divisões dos estudos e delimitações

As inclusões dos artigos foram feitas de forma independente por dois pesquisadores. A seleção dos artigos foi realizada em várias etapas: título do estudo, resumo e texto completo. Os critérios de exclusão foram: artigos de revisão bibliográfica, artigos duplicados, monografias, dissertações e teses.

Os resultados encontrados foram todos tabulados com os seguintes critérios: ano da publicação, base de dados encontrada, país, delineamento da pesquisa realizada, amostra da pesquisa, protocolos utilizados durante 0 treino muscular respiratório, dispositivos utilizados e conclusão do estudo. Depois da tabulação os levantamentos da pesquisa foram delimitados e relatados nos resultados e complementados na discussão.

\section{RESULTADOS}

Foram encontrados nas bases de dados padronizadas durante a pesquisa, 136 artigos com os descritores definidos no início da pesquisa (fluxograma 1).

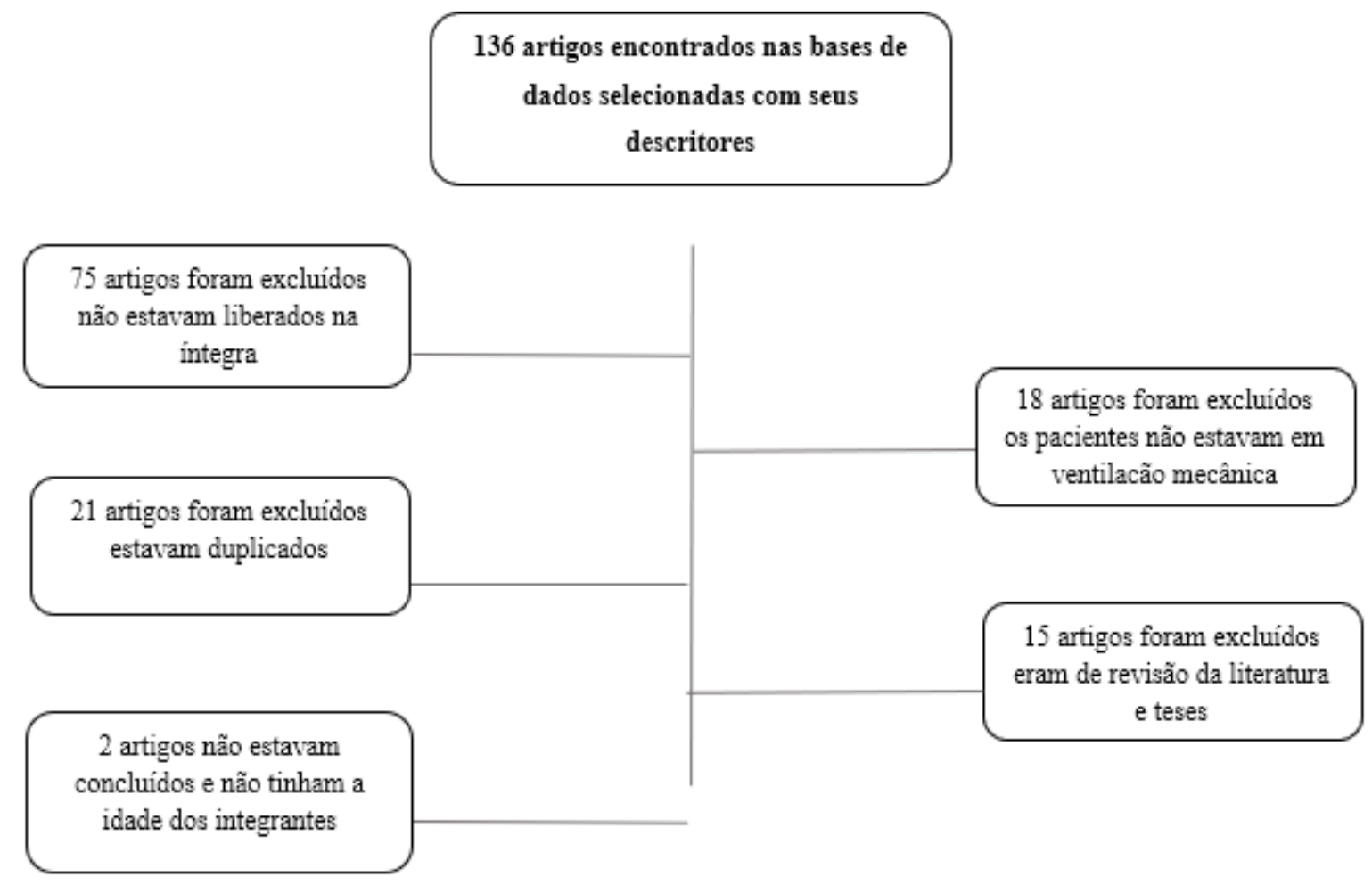

5 artigos incluídos após avaliação dos

critérios de inclusão na revisão

sistemática

Fluxograma 1. Delimitação da pesquisa e os resultados encontrados. 
Apenas cinco artigos incluídos na revisão, com rigor metodológico em todos os protocolos de treino muscular inspiratório e em todos não eram específicos para idosos, porém a idade média encontrada dos participantes estavam entre 60 e 81 anos nos estudos. Todos os indivíduos estavam em ventilação mecânica com desmame difícil (paciente em tubo orotraqueal com tempo superior a 7 dias) e prolongado (paciente com falha no teste de respiração espontânea por mais de 3 vezes) $)^{11}$, estáveis hemodinamicamente (quadro 1).

Os artigos apresentaram caráter de intervenção e foram randomizados (entre grupo controle e grupo intervenção), dentro de unidades de terapia intensiva ou domiciliar. Em todos os artigos foram relatadas variáveis que foram coletadas antes e após o treino muscular inspiratório, com desfecho dos treinos realizados nos pacientes idosos que estavam com tubo orotraqueal ou traqueostomizados.

Os protocolos identificados durante a pesquisa mostraram que no treino muscular inspiratório randomizado, os participantes eram separados em grupo de intervenção, grupo observacional e grupo controle, apenas um dos artigos foi realizado o relato de caso isolado de uma paciente.

Um estudo ${ }^{12}$ realizou em ambos os grupos sessões de fisioterapia respiratória diariamente durante sete dias da semana até o paciente sair do suporte ventilatório, com manobras de higiene brônquica e aspiração traqueal. As sessões de fisioterapia motora foram compostas por três sessões diárias, realizando dez repetições com exercícios ativo-assistidos e passivos. O grupo intervenção recebeu as sessões de fisioterapia convencional e uma vez ao dia de treinamento muscular respiratório (TMR), com o dispositivo Threshold IMT (Respironics), conectado diretamente na traqueostomia do paciente, as sessões eram compostas por três séries de dez repetições e com carga de $20 \%$ da primeira PImáx, com a cabeceira ficava elevada à $45^{\circ}$, mantendo um intervalo para descanso de dois minutos entre cada série.

Como também podemos observar em outro estudo ${ }^{13}$, o qual os dados corroboraram com o mesmo protocolo do estudo anterior, randomizando os grupos, em intervenção associada a fisioterapia convencional e o grupo controle que realizou apenas fisioterapia convencional. Ambos os grupos realizaram mobilização assistida, higiene brônquica e aspiração de traqueostomia com exercícios de membros superiores e inferiores. 0 que identificamos neste estudo que o grupo intervenção realizou o protocolo com auxílio do dispositivo o HS730, Respironics, New Jersey, EUA, este equipamento foi conectado direto na traqueostomia com intensidade da carga de $50 \%$ equivalente da primeira PImáx, aumentando gradativamente a intensidade máxima tolerável, com 5 series com 6 repetições cada e intervalo de descanso entre as series de 1 minuto.

Os protocolos relatados anteriormente, também são similares a outro estudo14, que randomizou os grupos que estavam em ventilação mecânica, porém com tubo orotraqueal, entre um grupo controle e intervenção. 0 grupo controle 
realizou a cada 12 horas, fisioterapia convencional com mobilização de membros, exercícios passivo e ativo-assistidos, higiene brônquica e aspiração do tubo orotraqueal, técnica do ciclo ativo da respiração (para pacientes cooperativos): é formada por três fases (técnica de respiração profunda, exercício de expansão torácica e huffing - técnica de expiração forçada). O grupo intervenção também realizou a fisioterapia convencional associada ao treino muscular inspiratório com o dispositivo Threshold®, Philips Respironics, Bruxelas Bélgica. Aplicou-se duas vezes ao dia, sendo a carga inicial de $9 \mathrm{~cm} \mathrm{H} \mathrm{H}_{2} \mathrm{O}$ com 4 séries de 6 a 8 respirações e cada sessão foi aumentada em $4 \mathrm{~cm} \mathrm{H}_{2} \mathrm{O}$ até o paciente ser extubado. Controverso aos dois estudos anteriores, que os pacientes estavam com traqueostomia, necessitando realizar mais sessões de treino muscular devido aos participantes estarem em desmame prolongado.

Já em outro estudo ${ }^{15}$, não foi realizado intervenção de fisioterapia motora entre os grupos, apenas foi delimitado em realizar ajustes pressóricos da sensibilidade do ventilador. Foram randomizados entre dois grupos, sendo que 0 grupo intervenção estava em ventilação mecânica com pressão de suporte que foi ajustada em um nível suficiente para atingir uma frequência respiratória de 20 a 30 respirações / min e volume corrente de 4 a $6 \mathrm{ml} / \mathrm{kg}$.

$\mathrm{O}$ suporte de pressão foi reduzido em $2 \mathrm{~cm} \mathrm{H}_{2} \mathrm{O}$ a cada hora para alcançar o suporte de pressão de $8 \mathrm{~cm}$ $\mathrm{H}_{2} \mathrm{O}$, eram realizadas duas sessões de exercício e foi baseado na diminuição gradual da sensibilidade do gatilho, para aumentar a resistência muscular assim a sensibilidade do gatilho foi ajustada em 20\% da primeira pressão inspiratória máxima (Pimáx), registrando no início do treinamento (na primeira sessão), o treinamento muscular inspiratório (TMI) foi limitado em 5 minutos, depois foi aumentado 5 minutos em cada sessão, até atingir 30 minutos. Se um paciente tolerasse 30 minutos de TMI, era classificado para o grupo intervenção e na próxima sessão era realizada o aumento da sensibilidade ao gatilho em $10 \%$ do PImáx inicial. Divergente dos demais artigos que todos utilizaram dispositivos de treino para auxiliar os protocolos durante o desmame.

Outro estudo ${ }^{16}$ realizou um relato de caso isolado de uma paciente idosa do sexo feminino com 77 anos, a paciente estava em ventilação domiciliar com desmame prolongado e o protocolo de intervenção foi realizar o treino muscular inspiratório com o dispositivo Powerbreathe® plus serie light, sendo realizadas por 2 vezes ao dia com 30 repetições, durante 4 semanas com carga linear graduada entre 50 e 60\% da pressão inspiratória máxima (PImáx), com reavaliação e ajuste de carga semanal associados a um programa de exercícios físicos ativo-assistidos e treino de marcha. 


\begin{tabular}{|c|c|c|c|c|c|}
\hline $\begin{array}{l}\text { BASE DE } \\
\text { DADOS E } \\
\text { AUTORES }\end{array}$ & $\begin{array}{c}\text { ANO DA } \\
\text { PUBLICAÇÃO, } \\
\text { PAÍS E TÍTULO }\end{array}$ & $\begin{array}{c}\text { DELINEAMENTO } \\
\text { DA PESQUISA }\end{array}$ & AMOSTRA & $\begin{array}{c}\text { PROTOCOLO } \\
\text { REALIZADO E } \\
\text { DISPOSITIVO } \\
\text { UTILIZADO }\end{array}$ & CONCLUSÃO \\
\hline $\begin{array}{l}\text { LILACS }^{(12)} \\
\text { PASCOTINI, } \\
\text { F.S. et al. }\end{array}$ & $\begin{array}{l}\text { 2014/ Brasil } \\
\text { Treinamento } \\
\text { muscular } \\
\text { respiratório } \\
\text { em pacientes } \\
\text { em desmame } \\
\text { da ventilação } \\
\text { mecânica. }\end{array}$ & $\begin{array}{c}\text { Pesquisa } \\
\text { experimental do } \\
\text { tipo pré e pós- } \\
\text { intervenção, com } \\
\text { análise quantitativa } \\
\text { dos dados e } \\
\text { traqueostomizados, } \\
\text { em processo } \\
\text { de desmame } \\
\text { da ventilação } \\
\text { mecânica. }\end{array}$ & $\begin{array}{c}\text { A amostra foi } \\
\text { constituída por } \\
14 \text { pacientes } \\
\text { predominantes } \\
\text { do sexo feminino } \\
\text { com idade entre } \\
60 \text { e } 81 \text { anos, } \\
\text { distribuídos } \\
\text { aleatoriamente } \\
\text { em Grupo } \\
\text { Controle } \\
\text { (GI) e Grupo } \\
\text { Experimental } \\
\text { (GII). }\end{array}$ & $\begin{array}{l}\text { Por sete dias, o } \\
\text { Grupo Controle } \\
\text { (GI) e o Grupo } \\
\text { Experimental } \\
\text { (GII) receberam } \\
\text { três sessões } \\
\text { de fisioterapia } \\
\text { respiratória, } \\
\text { composta por } \\
\text { manobras de } \\
\text { higiene brônquica e } \\
\text { aspiração traqueal, } \\
\text { e três sessões de } \\
\text { fisioterapia motora, } \\
\text { com o paciente em } \\
\text { decúbito dorsal, } \\
\text { composta por dez } \\
\text { repetições dos } \\
\text { seguintes exercícios } \\
\text { ativo-assistidos. } \\
\text { O Gll realizou, } \\
\text { adicionalmente } \\
\text { às três sessões } \\
\text { de fisioterapia } \\
\text { respiratória e motora, } \\
\text { o treino muscular } \\
\text { respiratório (TMR) } \\
\text { uma vez ao dia } \\
\text { com o dispositivo } \\
\text { Threshold IMT® } \\
\text { (Respironics). }\end{array}$ & $\begin{array}{c}\text { Observou-se aumento } \\
(\mathrm{p}=0,02)^{*} \text { na FR* } \mathrm{e} \\
\text { redução da PImáx ( } \mathrm{p}=0,04) \\
\text { no } \mathrm{Gl} \text {, demonstrando } \\
\text { aumento do trabalho } \\
\text { respiratório e perda de } \\
\text { força muscular entre o } \\
\text { primeiro e sétimo dia de } \\
\text { desmame. No GII, as } \\
\text { variáveis não sofreram } \\
\text { alterações significativas, } \\
\text { observando-se a } \\
\text { manutenção da função } \\
\text { respiratória. Concluindo-se } \\
\text { que, o TMR* foi benéfico, } \\
\text { garantindo a manutenção } \\
\text { dos parâmetros } \\
\text { respiratórios, podendo } \\
\text { ser um aliado para o } \\
\text { desmame. }\end{array}$ \\
\hline
\end{tabular}




\begin{tabular}{|c|c|c|c|c|c|}
\hline $\begin{array}{l}\text { BASE DE } \\
\text { DADOS E } \\
\text { AUTORES }\end{array}$ & $\begin{array}{c}\text { ANO DA } \\
\text { PUBLICAÇÃO, } \\
\text { PAÍS E TÍTULO }\end{array}$ & $\begin{array}{c}\text { DELINEAMENTO } \\
\text { DA PESQUISA }\end{array}$ & AMOSTRA & $\begin{array}{c}\text { PROTOCOLO } \\
\text { REALIZADO E } \\
\text { DISPOSITIVO } \\
\text { UTILIZADO }\end{array}$ & CONCLUSÃO \\
\hline $\begin{array}{c}\text { MEDLINE }{ }^{(14)} \\
\text { ELBOHY } \\
\text { MS, et al }\end{array}$ & $\begin{array}{c}\text { 2014/ Estados } \\
\text { Unidos } \\
\text { Effect of } \\
\text { respiratory } \\
\text { muscles training } \\
\text { in weaning of } \\
\text { mechanically } \\
\text { ventilated COPD } \\
\text { patients. }\end{array}$ & $\begin{array}{c}\text { O estudo } \\
\text { randomizado e } \\
\text { intervencionista } \\
\text { em pacientes com } \\
\text { DPOC* admitidos } \\
\text { na } \\
\text { unidade de terapia } \\
\text { intensiva no } \\
\text { hospital do coração } \\
\text { de Abbassia, no } \\
\text { período de outubro } \\
2012 \text { à } \\
\text { Março-2013. }\end{array}$ & $\begin{array}{l}\text { Os pacientes } \\
\text { foram } \\
\text { subdivididos } \\
\text { em } 2 \text { grupos: } \\
\text { GRUPO (A): } \\
\text { (20 pacientes) } \\
\text { incluem } \\
\text { pacientes que } \\
\text { receberam } \\
\text { treinamento e } \\
\text { GRUPO (B): } \\
\text { (20 pacientes) } \\
\text { incluem } \\
\text { pacientes que } \\
\text { não receberam } \\
\text { Treinamento. } \\
\text { Com predomínio } \\
\text { do sexo } \\
\text { masculino e } \\
\text { idade média } \\
\text { entre } 61 \text { e } 64 \\
\text { anos. }\end{array}$ & $\begin{array}{c}\text { Grupo (A) Estavam } \\
\text { em pressão de } \\
\text { suporte reduzido } \\
\text { em } 2 \mathrm{~cm} \mathrm{H}_{2} \mathrm{O} \text { a } \\
\text { cada hora para } \\
\text { alcançar o suporte } \\
\text { de pressão } 8 \mathrm{~cm} \\
\mathrm{H}_{2} \mathrm{O} \text {. Duas vezes ao } \\
\text { dia. O treinamento } \\
\text { foi baseado na } \\
\text { diminuição da } \\
\text { sensibilidade } \\
\text { do ventilador } \\
\text { gradualmente, a } \\
\text { fim de aumentar a } \\
\text { resistência muscular. } \\
\text { Grupo (B). Os } \\
\text { pacientes foram } \\
\text { desmamados } \\
\text { na modalidade } \\
\text { pressão de suporte } \\
\text { sem ajustar a } \\
\text { sensibilidade. }\end{array}$ & $\begin{array}{l}\text { Houve uma diferença } \\
\text { significativa entre os dois } \\
\text { grupos em relação aos } \\
\text { desfechos primários } \\
\text { incluindo taxa de sucesso } \\
\text { no desmame, duração } \\
\text { da ventilação mecânica, } \\
\text { tempo de internação } \\
\text { na unidade de terapia } \\
\text { intensiva e } \\
\text { hospitalar. Também houve } \\
\text { melhora significativa dos } \\
\text { desfechos secundários no } \\
\text { grupo (A) } \\
\text { incluindo PO }{ }_{2} \text {, saturação } \\
\text { de } \mathrm{O}_{2} \text { e pressão } \\
\text { inspiratória máxima ao } \\
\text { longo dos } 5 \text { dias de treino } \\
\text { muscular inspiratório; } \\
\text { enquanto houve uma } \\
\text { significativa } \\
\text { diferença entre os dois } \\
\text { grupos em relação aos } \\
\text { dificuldade de desmame. } \\
\text { desfechos secundários } \\
\text { acima em favor do grupo } \\
\text { (A). Concluíram que o } \\
\text { treinamento muscular } \\
\text { inspiratório aumenta } \\
\text { a força e a resistência } \\
\text { muscular, bem como desmame da } \\
\text { auilo mecânica em } \\
\text { peom }\end{array}$ \\
\hline
\end{tabular}




\begin{tabular}{|c|c|c|c|c|c|}
\hline $\begin{array}{l}\text { BASE DE } \\
\text { DADOS E } \\
\text { AUTORES }\end{array}$ & $\begin{array}{c}\text { ANO DA } \\
\text { PUBLICAÇÃO, } \\
\text { PAÍS E TÍTULO }\end{array}$ & $\begin{array}{c}\text { DELINEAMENTO } \\
\text { DA PESQUISA }\end{array}$ & AMOSTRA & $\begin{array}{l}\text { PROTOCOLO } \\
\text { REALIZADO E } \\
\text { DISPOSITIVO } \\
\text { UTILIZADO }\end{array}$ & CONCLUSÃO \\
\hline $\begin{array}{l}\text { LILACS }^{(16)} \\
\text { NEPOMU- } \\
\text { CENO } \\
\text { JUNIOR, } \\
\text { B. R. V. et } \\
\text { al. }\end{array}$ & $\begin{array}{l}\text { 2016/ Brasil } \\
\text { Treinamento } \\
\text { muscular } \\
\text { inspiratório } \\
\text { e desmame } \\
\text { ventilatório de } \\
\text { paciente com } \\
\text { síndrome pós- } \\
\text { pólio. }\end{array}$ & $\begin{array}{c}\text { O estudo relato } \\
\text { de caso de uma } \\
\text { paciente idosa na } \\
\text { ventilação mecânica } \\
\text { domiciliar. }\end{array}$ & $\begin{array}{c}\text { Idosa de sexo } \\
\text { feminino, } 77 \text { anos, } \\
\text { ficou internada } \\
\text { no hospital por } \\
60 \text { dias, devido a } \\
\text { IRPA*secundário } \\
\text { fraqueza muscular } \\
\text { e paresia } \\
\text { diafragmática à } \\
\text { esquerda associado } \\
\text { à síndrome pós- } \\
\text { poliomielite (SPP)*. } \\
\text { O diagnóstico da } \\
\text { SPP foi confirmado } \\
\text { por critérios } \\
\text { internacionais. } \\
\text { Na admissão } \\
\text { em domicílio, } \\
\text { paciente lúcida, } \\
\text { sob ventilação } \\
\text { mecânica (VM)* } \\
\text { domiciliar no } \\
\text { Trilogy } 100 \text { da } \\
\text { Respironics® via } \\
\text { traqueostomia } \\
\text { plástica, modo } \\
\text { espontâneo time } \\
\text { (ST)*, intolerante } \\
\text { aos modos } \\
\text { assistidos. }\end{array}$ & $\begin{array}{c}\text { Investiu-se no } \\
\text { treinamento muscular } \\
\text { inspiratório (TMI)* } \\
\text { com Powerbreathe® } \\
\text { plus, serie light por } \\
30 \text { repetições por } \\
2 \text { vezes ao dia, } \\
\text { durante } 4 \text { semanas, } \\
\text { associados a } \\
\text { um programa de } \\
\text { exercícios físicos. }\end{array}$ & $\begin{array}{c}\text { Concluiu-se que a } \\
\text { estratégia apresentou } \\
\text { desfecho do desmame } \\
\text { da ventilação } \\
\text { mecânica (VM)*, } \\
\text { assim como melhora } \\
\text { concomitante na } \\
\text { PImáx e a Mensuração } \\
\text { de Independência } \\
\text { Funcional (MIF)*, } \\
\text { retornando ao final } \\
\text { deste curto programa } \\
\text { de reabilitação a realizar } \\
\text { atividades laborais em } \\
\text { domicílio. }\end{array}$ \\
\hline
\end{tabular}




\begin{tabular}{|c|c|c|c|c|c|}
\hline $\begin{array}{l}\text { BASE DE } \\
\text { DADOS E } \\
\text { AUTORES }\end{array}$ & $\begin{array}{c}\text { ANO DA } \\
\text { PUBLICAÇÃO, } \\
\text { PAÍS E TíTULO }\end{array}$ & $\begin{array}{c}\text { DELINEAMENTO } \\
\text { DA PESQUISA }\end{array}$ & AMOSTRA & $\begin{array}{c}\text { PROTOCOLO } \\
\text { REALIZADO E } \\
\text { DISPOSITIVO } \\
\text { UTILIZADO }\end{array}$ & CONCLUSÃO \\
\hline $\begin{array}{l}\text { MEDLINE }{ }^{(13)} \\
\text { BISSETT, } \\
\text { B. M. et al. }\end{array}$ & $\begin{array}{l}\text { 2016/ Australia } \\
\text { Inspiratory } \\
\text { muscle training to } \\
\text { enhance recovery } \\
\text { from mechanical } \\
\text { ventilation: a } \\
\text { randomised trial. }\end{array}$ & $\begin{array}{l}\text { Ensaio randomizado } \\
\text { com análise de } \\
\text { cego-avaliador e } \\
\text { intenção de tratar. } \\
\text { Após } 48 \text { horas de } \\
\text { desmame bem- } \\
\text { sucedido. }\end{array}$ & $\begin{array}{c}70 \text { participantes } \\
\text { (ventilados } \\
\text { mecanicamente } \\
\geq 7 \text { dias) foram } \\
\text { randomizados para } \\
\text { receber treinamento } \\
\text { muscular } \\
\text { inspiratório uma } \\
\text { vez ao dia, } 5 \text { dias } \\
\text { / semana por } 2 \\
\text { semanas, além dos } \\
\text { cuidados habituais } \\
\text { ou cuidados } \\
\text { habituais (controle). } \\
\text { Foram } \\
\text { selecionados } \\
\text { após } 48 \text { horas } \\
\text { de desmame } \\
\text { bem-sucedido, } \\
\text { traqueostomizados. } \\
\text { A maioria dos } \\
\text { indivíduos com } \\
\text { díade acima de } \\
60 \text { anos. Um } \\
\text { grupo recebeu } \\
\text { o treinamento } \\
\text { muscular } \\
\text { inspiratório uma } \\
\text { vez ao dia, } 5 \text { dias } \\
\text { / semana por } 2 \\
\text { semanas, além dos } \\
\text { cuidados habituais } \\
\text { ou cuidados } \\
\text { habituais. }\end{array}$ & $\begin{array}{l}\text { Os participantes } \\
\text { foram randomizados } \\
\text { para receber os } \\
\text { cuidados habituais } \\
\text { (grupo controle) } \\
\text { ou dispositivo de } \\
\text { treino HS730, } \\
\text { Respironics, New } \\
\text { Jersey, EUA (IMT)*, } \\
\text { além de cuidados } \\
\text { habituais (grupo } \\
\text { IMT) }{ }^{*} \text { por } 2 \text { semanas } \\
\text { após a inscrição. } \\
\text { A fisioterapia de } \\
\text { cuidados usuais } \\
\text { incluiu um programa } \\
\text { de intervenções } \\
\text { individualizado e } \\
\text { supervisionado, que } \\
\text { incluiu qualquer } \\
\text { um dos seguintes: } \\
\text { mobilização } \\
\text { assistida, } \\
\text { tratamentos } \\
\text { de limpeza de } \\
\text { secreções incluindo } \\
\text { técnicas de pressão } \\
\text { expiratória positiva, } \\
\text { exercícios de } \\
\text { respiração profunda } \\
\text { sem dispositivo } \\
\text { de resistência } \\
\text { e exercícios de } \\
\text { membros superiores } \\
\text { e inferiores. }\end{array}$ & 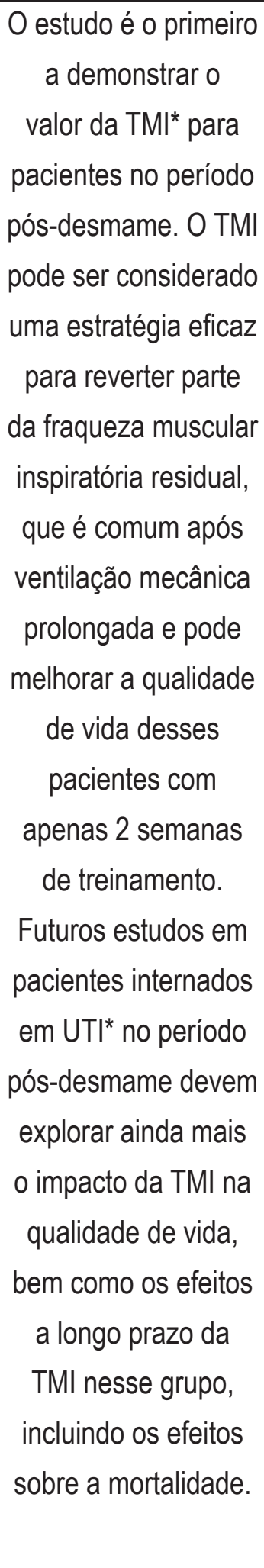 \\
\hline
\end{tabular}




\begin{tabular}{|c|c|c|c|c|c|}
\hline $\begin{array}{l}\text { BASE DE } \\
\text { DADOS E } \\
\text { AUTORES }\end{array}$ & $\begin{array}{c}\text { ANO DA } \\
\text { PUBLI- } \\
\text { CAÇÃO, } \\
\text { PAíS E } \\
\text { TíTULO }\end{array}$ & $\begin{array}{c}\text { DELINEAMENTO } \\
\text { DA PESQUISA }\end{array}$ & AMOSTRA & $\begin{array}{l}\text { PROTOCOLO } \\
\text { REALIZADO E } \\
\text { DISPOSITIVO } \\
\text { UTILIZADO }\end{array}$ & CONCLUSÃO \\
\hline $\begin{array}{l}\text { PUBMED }{ }^{(16)} \\
\text { NAFAE, R. } \\
\text { et al }\end{array}$ & $\begin{array}{l}\text { 2018/Egito } \\
\text { Effect of } \\
\text { multimodal } \\
\text { physiotherapy } \\
\text { on outcome of } \\
\text { mechanically } \\
\text { ventilated } \\
\text { patients } \\
\text { at zagazig } \\
\text { university } \\
\text { respiratory } \\
\text { intensive care } \\
\text { unit in (2014- } \\
\text { 2015). }\end{array}$ & $\begin{array}{c}\text { Estudo } \\
\text { randomizado com } \\
\text { grupo controle em } \\
\text { pacientes intubados } \\
\text { após apresentarem } \\
\text { quadro de } \\
\text { insuficiência } \\
\text { respiratória aguda } \\
\text { na Unidade de } \\
\text { Terapia Intensiva } \\
\text { Respiratória do } \\
\text { Zagazig } \\
\text { Hospitais } \\
\text { Universitários } \\
\text { durante um período } \\
\text { de } 2 \text { anos } \\
\text { (maio de 2014 a } \\
\text { abril de 2016). }\end{array}$ & $\begin{array}{c}\text { A idade } \\
\text { média dos } \\
\text { indivíduos era } \\
\text { de } 60 \text { anos } \\
\text { predominante } \\
\text { do sexo } \\
\text { feminino. Com } \\
\text { total de } 40 \\
\text { participantes } \\
\text { sendo } 20 \\
\text { no grupo } \\
\text { intervenção e } \\
20 \text { no grupo } \\
\text { controle. }\end{array}$ & $\begin{array}{l}\text { O Grupo de controle: } \\
\text { Realiza uma vez ao dia } \\
\text { fisioterapia com: } \\
\text { 1) Mobilização } \\
\text { • Postural; } \\
\text { • Exercícios passivo e } \\
\text { ativo. } \\
\text { Higiene brônquica e } \\
\text { exercício e huffing } \\
\text { "Expiratório forçado } \\
\text { técnical). } \\
\text { O grupo de intervenção: } \\
\text { Foram realizados } \\
\text { treinamento muscular } \\
\text { inspiratório } \\
\text { (IMT) } \\
\text { Com o } \\
\text { dispositivo. Threshold® } \\
\text { IMT* } \\
\text { Philips Respironics, } \\
\text { Bruxelas, Bélgica. } \\
\text { Foi realizado o protocolo } \\
\text { duas vezes ao dia até o } \\
\text { paciente ser extubado. } \\
\text { Com carga inicial de } \\
9 \text { cm } \mathrm{H}_{2} \mathrm{O} \text { e em cada } \\
\text { sessão era realizada } \\
4 \text { séries de } 6-8 \\
\text { respirações e cada } \\
\text { sessão foi aumentada } \\
\text { em } 4 \text { cm } \mathrm{H}_{2} \mathrm{O} \text { e } 5 \text { min } \\
\text { em duração máxima } 30 \\
\text { min. }\end{array}$ & $\begin{array}{l}\text { Fisioterapia convencional } \\
\text { na Unidade de terapia } \\
\text { intensiva é segura por ter } \\
\text { intervenção com poucas } \\
\text { complicações; também } \\
\text { isso } \\
\text { resulta em mudanças } \\
\text { positivas no } \\
\text { pressão inspiratória para } \\
\text { mecanicamente } \\
\text { pacientes com } \\
\text { insuficiência respiratória } \\
\text { ventilada. Já os pacientes } \\
\text { que realizaram o } \\
\text { treinamento muscular } \\
\text { inspiratório e } \\
\text { convencional } \\
\text { fisioterapia apresentaram } \\
\text { melhora pode melhorar } \\
\text { da pressão inspiratória } \\
\text { máxima, capacidade } \\
\text { funcional e } \\
\text { Tobin e redução da } \\
\text { de terapia intensiva. }\end{array}$ \\
\hline
\end{tabular}

Quadro 1. Descrição dos artigos incluídos na pesquisa. ${ }^{*}$ TMR: Treino muscular respiratório; FR: frequência respiratória; DPOC: doença pulmonar obstrutiva crônica; TMI: treino muscular inspiratório; UTI: unidade de terapia intensiva; VM: ventilação mecânica; MIF: Mensuração de Independência Funcional. 


\section{DISCUSSÃO}

Esta revisão sistemática da literatura analisou os artigos encontrados sobre o treino muscular inspiratório em idosos na ventilação mecânica. Está analise verificou os principais protocolos de treino muscular utilizados nos últimos 5 anos e os dispositivos empregados. Avalidação metodológica foi rigorosa, excluindo os artigos que não foram enquadrados em nossa revisão e os principais dados encontrados mostraram que $80 \%$ dos estudos não foram específicos para idosos, porém os participantes incluídos apresentaram idade acima de 60 anos e em suporte ventilatório.

Todos utilizaram protocolos com dispositivos de carga linear ou fixa, porém um estudo ${ }^{15}$ realizou ajuste de sensibilidade e se o paciente não tolerasse o protocolo era colocado no grupo controle, que não realizou nenhum tipo de treinamento, devido aos pacientes não toleravam pressões baixas na ventilação mecânica apresentando instabilidade hemodinâmica durante o treino que foi interrompido. Estes pacientes foram apenas observados durante a evolução do desmame ventilatório. Porém esta técnica de ajuste da sensibilidade vem apresentando contraindicação desde de 2013, devido não estar próximo da sensibilidade fisiológica do paciente ${ }^{17}$.

Em todos os estudos foram observados aumentos significativos da PImáx após o treino muscular inspiratório. Entretanto, os idosos do grupo controle, apresentaram manutenção dos valores da PImáx com protocolo de fisioterapia convencional. Apenas um estudo ${ }^{16}$ não realizou randomização devido ser um relato de caso.

APImáx foi mensuradas através do equipamento Manovacuômetro, que identificou a pressão inspiratória durante a oclusão da válvula unidirecional, podendo ser realizada com 20 até 40 segundos de oclusão na inspiração ${ }^{18}$. Este teste foi realizado 3 vezes para cada medida e o melhor valor foi agregado para todos os estudos tanto nos valores iniciais quanto finais de treino, porém os valores de oclusão foram variáveis entre 20, 25 e 40 segundos.

No protocolo do treino muscular inspiratório de um estudo ${ }^{19}$, o dispositivo Threshold IMT囚 foi utilizado em pacientes com tubo orotraqueal com processo de desmame da ventilação mecânica, com carga e aumento da PImáx no grupo intervenção. Quatro dos estudos selecionados encontraram o aumento da PImáx, apenas no estudo de Pascotine et al 2014, não apresentou aumento em relação ao grupo controle.

Outro dispositivo de TMI foi utilizado em uma pesquisa ${ }^{20}$. 0 estudo comparou o treino muscular inspiratório com dispositivo Powerbreathe® KH2 (IMT Technologies Ltd, Birmingham, Inglaterra) com o grupo controle (nebulização e fisioterapia convencional). 0 resultado mostrou que o grupo de treinamento muscular inspiratório com o dispositivo Powerbreathe $®$, apresentou aumento significativo da PImáx.

Em relação ao tempo de desmame, um estudo realizado ${ }^{12}$, que apesar de não ser realizado com idosos, não apresentou diferença de dias de desmame ventilatório entre os grupos controle e intervenção, diferentemente dos 
demais artigos desta revisão que apresentaram um tempo de desmame menor em relação ao grupo controle. Outro estudo ${ }^{21}$ vai ao encontro dos artigos selecionados, que realizou o uso de um dispositivo de carga linear com 40\% da PImáx em idosos com mais de 65 anos. Sendo assim, mostrando segurança e a importância do treino muscular inspiratório em idosos auxiliando no desmame da ventilação mecânica.

Em uma revisão sistemática ${ }^{22}$, foi observado redução do índice de Tobin, aumento da Plmáx e menor tempo de desmame da ventilação mecânica, corroborando com os estudos investigados em nossa pesquisa. Entretanto, outra revisão da literatura, como no ${ }^{23}$, relatou que o treino muscular inspiratório evidenciou aumento da PImáx após o protocolo, porém não ocorrendo o sucesso do desmame dos pacientes que realizaram a intervenção.

Mesmo diante dos interessantes resultados apresentados nesta revisão, a mesma possui algumas limitações a serem apresentadas: a falta de inclusão de estudos de outras línguas, como o espanhol; a escassez de estudos na área, principalmente com idosos.

\section{CONSIDERAÇÕES FINAIS}

A presente revisão sistemática evidenciou a importância do treino muscular inspiratório em idosos auxiliando no desmame da ventilação mecânica, porém, faz-se necessária a realização de novos estudos que busquem identificar diferença de dias de desmame ventilatório entre os grupos controle e intervenção.

\section{REFERÊNCIAS}

1. Gottlieb MGV, Schwanke CHA, Gomes I, Cruz IBM. Envelhecimento e longevidade no Rio Grande do Sul: um perfil histórico, étnico e de morbi-mortalidade dos idosos. Rev Bras Geriatr e Gerontol 2011;14(2):365-80.

2. Ruivo S, Viana P, Martins C, Baeta C. Efeito do envelhecimento cronológico na função pulmonar. Comparação da função respiratória entre adultos e idosos saudáveis. Rev Port Pneumol. 2009;15(4):629-53.

3. Mazullo Filho JBR, Bona S, Pase D, Alberto L, Junior F, Dias AS. The effects of mechanical ventilation on oxidative stress. Rev Bras Ter Intensiva. 2012;24(2):23-9. 
4. Fonseca NM, Martins AVC, Fonseca GG. Lung protective ventilation (PPV): can we use for all patient? Rev Médica Minas Gerais. 2014;24(Supl 8):67-72.

5. Fonseca M de A, Cader SA, Dantas EHM, Bacelar SC, Silva EB da, Leal SM de O. Programas de treinamento muscular respiratório: impacto na autonomia funcional de idosos. Rev Assoc Med Bras. 2010;56(6):642-8.

6. Costa R, Carolina A. Fatores que influenciam no sucesso do desmame da ventilação mecânica a partir da suspensão da sedação A Ventilação Mecânica ( VM ) é a modalidade mais utilizada para suporte de vida na Insuficiência. 2012;4(2):19-25.

7. Cunha CS, Rodrigues E, Santana M, Fortes RA. Técnicas de Fortalecimento da Musculatura Respiratória Auxiliando o Desmame do Paciente em Ventilaçao Mecânica Invasica. Cad UniFOA. 2008;06:80-6.

8. Bissett BM, Leditschke IA, Paratz JD, Boots RJ. Protocol: Inspiratory muscle training for promoting recovery and outcomes in ventilated patients (IMPROVe): A randomised controlled trial. BMJ Open. 2012;2(2):1-8.

9. Ercole FF, Melo LS de, Alcoforado CLGC. Integrative review versus systematic review. Reme Rev Min Enferm. 2014;18(1):9-11.

10. Itens P, Revis R, Uma P. Principais itens para relatar Revisões sistemáticas e Meta-análises: A recomendação PRISMA. Epidemiol e Serviços Saúde [Internet]. 2015;24(2):335-42.

11. Barba. Diretrizes brasileiras de ventilação mecânica - 2013. AMIB. 2013;

12. Pacotine $\mathrm{F} \mathrm{e} \mathrm{C}$ et al Treinamento muscular respiratório em pacientes em desmame da ventilação mecânica. ABCS Heal Sci. 2014;39(3):133-41.

13. Bissett BM, Leditschke IA, Neeman T, Boots R, Paratz J. Inspiratory muscle training to enhance recovery from mechanical ventilation: A randomised trial. Thorax. 2016;71(9):812-9.

14. Nafae RM, El-shahat HM, Shehata SM, Zaki LG. Effect of multimodal physiotherapy on outcome of. 
ZUMJ. 2018;24(3):178-91.

15. Elbouhy MS, AbdelHalim HA, Hashem AMA. Effect of respiratory muscles training in weaning of mechanically ventilated COPD patients. Egypt J Chest Dis Tuberc. 2014;63(3):679-87.

16. Nepomuceno Júnior, BRV, et al. Treinamento muscular inspiratório e desmame ventilatório de paciente com síndrome pós-pólio. Fisioter Brasil. 2016;17(2):126-129.

17. Jerre G. et al. Fisioterapia no paciente sob ventilação mecânica. J Bras Pneumol . 2017;33(suppl 2):14250 ;

18. Ferreira $A G$ et al. Comparação entre a pressão inspiratória máxima aferida pelo manovacuômetro digital e pelo dispositivo eletrônico de treinamento muscular inspiratório. Sci Med (Porto Alegre). 2016;26(4):4-11.

19. Dixit A, Prakash S. Effects of Threshold Inspiratory Muscle Training Versus Conventional Physiotherapy on the Weaning Period of Mechanically Ventilated Patients: a Comparative Study. Int J Physiother Res. 2014;2(2):424-8.

20. Tonella RM et al. Inspiratory Muscle Training in the Intensive Care Unit: A New Perspective. J Clin Med Res. 2017;9(11):929-34.

21. Condessa RL, Brauner JS, Saul AL, Baptista M, Silva ACT. Inspiratory muscle training did not accelerate weaning from mechanical ventilation but did improve tidal volume and maximal respiratory pressures : a randomised trial. Aust J Physiother. 2013;59(2):101-7.

22. Jaenisch RB SJ. Efeito do treinamento muscular inspiratório no desmame e extubação de pacientes em ventilação mecânica: uma revisão de literatura. 2017; 2 (2): 85-95.

23. Patsaki I, Papadopoulos E, Sidiras G, Christakou A, Kouvarakos A, Markaki V. The Effectiveness of Inspiratory Muscle Training in Weaning Critically III Patients from Mechanical Ventilation. Hosp Chronicles. 2013;8(2):86-90. 